\title{
Spatial localization in saccade and pursuit-eye-movement conditions: A comparison of perceptual and motor measures
}

\author{
HITOSHI HONDA \\ Nigata University, Niigata, Japan
}

\begin{abstract}
The ability to localize the spatial position of a visual target fixated by a saccade or a pursuit eye movement was examined using motor and perceptual measures. When subjects were asked to reproduce the distance of the target displacement (perceptual measure), the produced distance in the pursuit-eye-movement conditions was shorter than that in the saccade conditions. The underestimation of the target displacement in the pursuit-eye-movement conditions was also found when subjects pointed manually to the position of the just extinguished visual target (motor measure) from the original starting position of the moving target. However, when the starting position of the pointing hand was varied from trial to trial, no difference in pointing performance was found between the two eye-movement conditions. The results provide clear evidence that there is a substantial difference between motor and perceptual localization of the visual target fixated by a saccade or a pursuit eye movement.
\end{abstract}

Rashbass (1961) reported evidence that a saccade is stimulated by the position of the visual target, whereas a pursuit eye movement is stimulated by the direction of movement and the velocity of the target. This suggests that the oculomotor system for saccades has more accurate information about the spatial location of the target than it does for pursuit eye movements. Festinger and Canon (1965) examined this hypothesis by asking subjects to perform a task involving eye-hand open-looped pointing to a visual stimulus presented in the dark, and found that the pointing performance was better when the subjects fixated the target by a saccade than when they tracked the moving target by a pursuit eye movement. The result was interpreted as indicating that the oculomotor system for saccades can provide accurate eye-position signals (EPSs), that is, information about the position of the eye within the orbit. A similar finding was reported by Honda (1984b) recently.

That less information about the eye position is available during pursuit eye movement has been shown by many studies using perceptual measures, too (Festinger \& Easton, 1974; Mack \& Herman, 1972; Stoper, 1973).

On the other hand, some recent studies have shown that subjects can accurately localize the target presented during a pursuit eye movement if a ballistic arm movement is used as a measure of an EPS, indicating that a fairly

This research was in part supported by a 1984 Grant-in-Aid for Scientific Research (No. 59510052) from the Ministry of Education, Science and Culture to the author.

Requests for reprints should be sent to Hitoshi Honda, Department of Psychology, Faculty of Humanities, Niigata University, Ikarashi, Niigata-shi, Niigata 950-21, Japan. accurate EPS is present during both saccades and smooth pursuit eye movements (Hansen, 1979; Hansen \& Skavenski, 1977).

To examine the above-mentioned discrepancy, the present study was designed to investigate the accuracy of EPS in saccade and pursuit-eye-movement conditions using both motor and perceptual measures.

\section{METHOD}

\section{Subjects}

Seven right-handed male university students (19-20 years of age) served as subjects. Two subjects had a slight myopia and wore spectacles during the experiment.

\section{Apparatus and Procedure}

The apparatus used in this study was the same as that used in Honda (1984a). A subject, seated in total darkness facing the apparatus for stimulus presentation, was asked to fix his head tightly in the forehead-and chinrest. A He-Ne laser bean (JLH-R2OS, Nihon Lasers) projected on a black screen through a mirror mounted on a galvanometer produced the visual stimuli in saccade and pursuiteye-movement conditions. A half mirror was set before the subject's eyes, and he looked at the optical image of the laser spot ( $2 \mathrm{~mm}$ in diameter) projected in front of him. A stimulus presented at the center of the visual field was used as a fixation point, which the experimenter was able to move horizontally at any speed by driving the galvanometer. Visual stimuli for eye movements were presented in the following two conditions: (1) In the saccade condition, 10 msec after the offset of the fixation point, a target visual stimulus was presented $12^{\circ}, 14^{\circ}$, or $16^{\circ}$ to the right of the fixation point. (2) In the pursuit-eye-movement condition, a visual stimulus started to move from the fixation point toward the right at a velocity of about $3 \% \mathrm{sec}$, and stopped at one of the above-mentioned three target positions.

The subject judged the location of the target in one of the following three response conditions. 


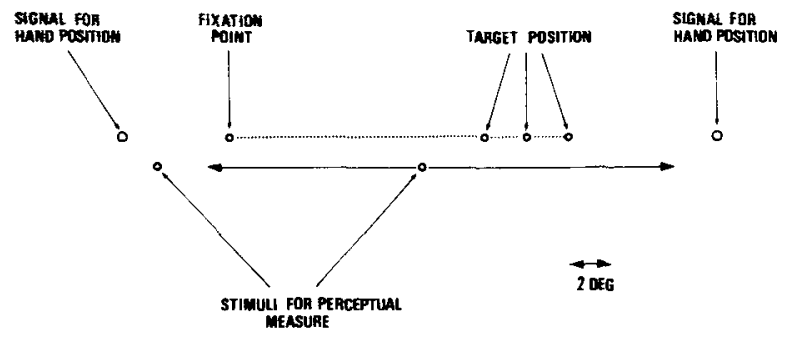

Figure 1. Relative locations of the fixation point, the target positions, the signals for positions of the pointing hand, and the visual stimuli for perceptual measures. A dotted line indicates the path of the target displacement in the pursuit-eye-movement conditions. A horizontal line with arrows indicates the extent of movement of the LED used for the perceptual measure.

(1) In Condition M1 (manual pointing from the fixation point), a buzzer warning signal ( $2 \mathrm{sec}$ in duration) was given, and then the fixation point was presented for 2 sec. The subject was asked to keep watching the fixation point and to place the index finger of his unobservable right hand on a movable fingerrest located at the position of the projected fixation point. The fingerrest was mounted on a rail placed along the path of the target displacement; it moved only in a horizontal direction. Immediately after the offset of the fixation point, the target stimulus was presented in either the saccade or the pursuit-eye-movement condition. The target was presented at the target position for $1 \mathrm{sec}$. After the offset of the target, the subject was allowed to point by moving the tip of the index finger of his right hand to where the target had been. The subject was asked to keep watching the target position while pointing. The tip of the index finger was set in a small hole $(1.5 \mathrm{~cm}$ in diameter) at the center of the fingerrest, and when the subject pointed to the target, the fingerrest moved with the finger. A needle attached to the fingerrest was used as an indicator of the finger position. The position of the index finger was measured by recording the position of the needle with a video-camera system. The distance between the target position and the tip of the index finger was measured to within $2 \mathrm{~mm}$. When a warning buzzer for the next trial was presented, the subject returned the finger to the original starting position, that is, the position of the fixation point. A small metal tack was attached at the starting position so that he could locate the position by touching the tack with the tip of his index finger.

(2) In Condition M2 (manual pointing from the right and left sides), the procedure was the same as that of Condition M1, except for the following modification: At the start of each trial, a smal] yellow LED, located to the right or left of the visual field, was repetitively turned on and off five times to indicate the starting position of the pointing hand. When the right LED, located $23^{\circ}$ to the right of the fixation point, was turned on and off, the subject moved the pointing hand to the right side, and when the left LED, located $5^{\circ}$ to the left of the fixation point, was turned on and off, he moved the pointing hand to the left side. The starting position of the pointing hand was about $25^{\circ}$ to the right or $7^{\circ}$ to the left of the fixation point. As in Condition M1, a metal tack was placed at each starting position of the hand so that the subject could locate the position with the hand. Two seconds after cessation of the repetitive lighting of the yellow LED, a warning signal and a visual target were presented. The subject made a saccade or a pursuit eye movement, and after the offset of the visual target, he pointed to the target position from the right side or from the left.

The reason for adopting two types of pointing condition was as follows: In Condition $\mathrm{M} 1$, in which pointing was done from the position of the fixation point, the starting position and the direction of the pointing movement were the same as those of the target movement. In that condition, the subject could point to the target position by moving the pointing hand to the perceived extent of the target movement. Thus, the subject might point to the target position on the basis of apparent distance of target displacement instead of pointing to the position where the target had disappeared, that is, to the position where the subject was looking. To avoid this kind of perceptual contamination, the starting position of the pointing hand in Condition M2 was varied from trial to trial. It was expected that in Condition M2 the subject could not use the apparent distance of the target displacement as a cue for pointing, especially when he pointed from the right side.

(3) In Condition P (perceptual reproduction of the distance of the target displacement), after a warning signal and the presentation " of a visual stimulus, the subject made a saccade or a pursuit eye movement to the targti, as in Condition Ml. Two seconds after the offset of the target, two red LEDs, which were used as stimuli for perceptual judgment of the distance of the target displacement (Figure 1), came on for $6 \mathrm{sec}$. The red LED located on the left side was stationary, whereas the horizontal position of the other red LED could be varied by about $2 / 3^{\circ}$ steps by rotating a knob located in front of the subject. The subject's task was to reproduce the distance of the target displacement by adjusting the interval between the two red LEDs.

Each subject was given four blocks of 24 trials for each response condition. In each block, 4 trials for each of six combinations of three target positions and two eye-movement conditions, a total of 24 trials, were given in random sequence. The interval between trials was about $7 \mathrm{sec}$. There was a rest period of about $10 \mathrm{~min}$ between blocks.

The subject's horizontal eye movements were monitored by a photoelectric apparatus, which recorded the movements of the right eye with an accuracy to at least $1^{\circ}$ for a $20^{\circ}$ rotation of the eye to the right of central gaze.

After completion of the experiment, we conducted a supplementary experiment in which the accuracy of pointing to a visible target on an illuminated background was examined. The procedure of the supplementary experiment was about the same as that of Condition M2, but the following modification was introduced. A scale was mounted horizontally across the black screen and illuminated by a small lamp. The fixation point and visible stimuli for saccades were presented on the scale. Each visual stimulus was presented at the target position for $7 \mathrm{sec}$, and the subject was asked to point to the target during its presentation with the unobservable hand. Thus, the subject was able to know the physical position of the target in the structured visual field. Six subjects who participated in the main experiment were used again as subjects. Each subject was given 10 trials for each target position, a total of 30 trials, in random sequence.

\section{RESULTS}

\section{Eye Movements}

The subject's eye movements, drawn on the recording chart by a large-amplitude recticoder (RJG-4024, Nihon Kohden) at a speed of $10 \mathrm{~mm} / \mathrm{sec}$, were analyzed by visual inspection.

Out of a total of 1,008 saccade trials for seven subjects, analysis of eye movements was impossible on 44 trials $(4.4 \%)$ because of such contaminations as eye blinking, and on 46 trials $(4.6 \%$ ) because the subjects failed to make a saccade, they tracked the target by multiple saccades that were sometimes interrupted by a small drift, or the latency of the saccade was extraordinarily large. These saccade trials were excluded from the data analysis of pointing performance and perceptual reproduction. On 125 of the remaining 918 saccade trials, the subjects showed a corrective saccade, whereas on 793 trials they fixated the target by a single saccade. 


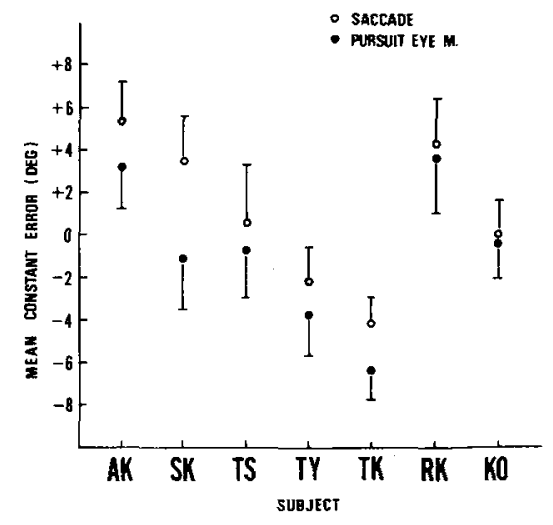

Figure 2. Mean constant error and variable error in Condition M1.

In the pursuit-eye-movement conditions, 80 (7.9\%) of 1,008 trials were excluded from the data analysis because of recording failures or because of interruptions by large saccades of more than $2^{\circ}$. The subjects sometimes showed a small saccade (108 trials) or a small drift (128 trials) during a pursuit eye movement. We used these trials for the data analysis of pointing and perceptual reproduction, because the size of these saccades and drifts was rather small (usually less than $1^{\circ}$ ) as compared with the extent of target displacement.

\section{Pointing Performance}

Condition M1. The distance between the position of the projected target and that of the pointing finger was measured on each trial, and the mean of the constant error (CE) and the variable error (VE), that is, the SD of the CEs, was calculated for each subject.

Figure 2 shows the mean CEs and the VEs in the two eye-movement conditions, separately for each subject. As shown in Figure 2, the subjects showed a constant directional bias in their pointing, that is, each subject tended to overshoot or undershoot the target position. Despite the constant directional bias, however, the position to which all subjects pointed was farther from the fixation point in the saccade conditions than in the pursuit-eyemovement conditions. According to statistical analysis, five of the seven subjects showed a significant difference in CEs between the two eye-movement conditions $[\mathrm{t}(60-94) \geq 4.29, \mathrm{p}<.01$, for Subjects A.K., T.K., T.Y., and S.K., and $t(66)=2.13, p<.05$, for Subject T.S.]. The vertical bars in Figure 2 show the VEs. The VEs were not significantly different between the two eyemovement conditions for any subjects.

Condition M2. Figure 3 shows the results of Condition M2. As in Condition M1, the subjects showed a large constant directional bias. A two-factor ANOVA (eye movement conditions $\times$ starting position of the pointing hand) was applied to the CE data of each subject. The results are summarized in Table 1 . The effect of eyemovement condition was significant only for Subjects S.K. and T.K. In these two subjects, the interaction of eye- movement conditon and starting position of the pointing hand was significant. The effect of the position of the pointing hand was significant for five subjects. Their pointing tended to show a bias toward the starting position. That is, when the subjects pointed from the right side, their pointing shifted somewhat to the right as compared with the pointing from the left.

Next, we analyzed the effects of the eye-movement condition, separately for the two starting positions. When they pointed from the left side, three subjects showed a significant difference in CEs between the two eye-movement conditions. On the other hand, when the subjects pointed from the right side, the effect of eye-movement conditon was not significant for any subjects (Table 1 and Figure $3 b$ ).

The vertical bars in Figure 3 show the VEs. A significant difference in VEs between the two eye-movement conditions was shown only for Subject R.K. [F(46,45) $=1.956, \mathrm{p}<.05$ ], who showed a large VE in the pursuiteye-movement condition. Next, the VEs between the two conditions of starting position of pointing were compared. A significant difference was shown for only two subjects. However, one subject (K.O.) showed a large VE when he pointed from the right side $[\mathrm{F}(44,44)=2.20, \mathrm{p}<$ .05], whereas another subject (S.K.) showed a large VE when he pointed from the left side $[\mathrm{F}(41,46)=1.90, \mathrm{p}<$ $.05]$. Thus, there was no consistent tendency in the VE data.
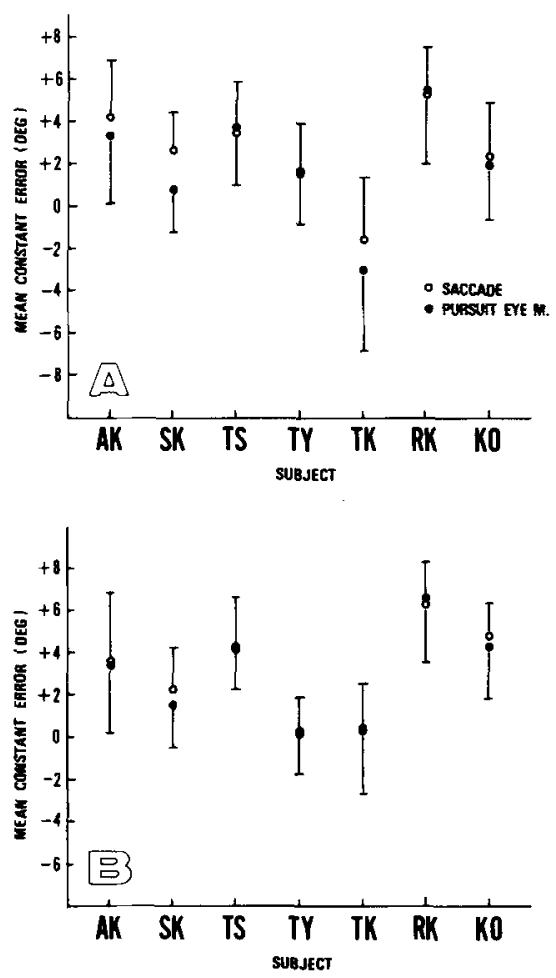

Figure 3. (A) Mean constant error and variable error in Condition M2. (B) Mean constant error and variable error when the subjects pointed from the right side in Condition $\mathrm{M2}$. 
Table 1

Summary of ANOVA for the CEs in Condition M2

\begin{tabular}{|c|c|c|c|c|c|}
\hline \multirow[b]{3}{*}{ Subject } & \multicolumn{5}{|c|}{ Source of Variation } \\
\hline & \multirow{2}{*}{\multicolumn{2}{|c|}{$\begin{array}{l}\text { Starting } \\
\text { Position of } \\
\text { the Hand }\end{array}$}} & \multirow[b]{2}{*}{ Interaction } & \multicolumn{2}{|c|}{ Eye-Movement Condition } \\
\hline & & & & $\begin{array}{l}\text { Pointing from } \\
\text { the Right }\end{array}$ & $\begin{array}{l}\text { Pointing from } \\
\text { the Left }\end{array}$ \\
\hline A.K. & n.s. & n.s. & n.s. & n.s. & $\begin{array}{c}p<.05 \\
F(1,91)=3.905\end{array}$ \\
\hline S.K. & $\begin{array}{c}\mathrm{p}<.01 \\
\mathrm{~F}(1,85)=22.26\end{array}$ & n.s. & $\begin{array}{c}\mathrm{p}<.01 \\
\mathrm{~F}(1,85)=6.05\end{array}$ & n.s. & $\begin{array}{c}p<.01 \\
F(1,85)=25.76\end{array}$ \\
\hline T.S. & n.s. & $\begin{array}{c}\mathrm{p}<.05 \\
\mathrm{~F}(1,86)=5.91\end{array}$ & n.s. & n.s. & n.s. \\
\hline T.Y. & n.s. & $\begin{array}{c}\mathrm{p}<.01 \\
\mathrm{~F}(1,88)=104.1\end{array}$ & n.s. & n.s. & n.s. \\
\hline T.K. & $\begin{array}{c}\mathrm{p}<.01 \\
\mathrm{~F}(1,89)=11.37\end{array}$ & $\begin{array}{c}\mathrm{p}<.01 \\
\mathrm{~F}(1,89)=142.3\end{array}$ & $\begin{array}{c}\mathrm{p}<.01 \\
\mathrm{~F}(1,89)=9.41\end{array}$ & n.s. & $\begin{array}{c}p<.01 \\
F(1,89)=20.72\end{array}$ \\
\hline R.K. & n.s. & $\begin{array}{c}\mathrm{p}<.01 \\
\mathrm{~F}(1,87)=15.87\end{array}$ & n.s. & n.s. & n.s. \\
\hline K.O. & n.s. & $\begin{array}{c}p<.01 \\
F(1,86)=165.1\end{array}$ & n.s. & n.s. & n.s. \\
\hline
\end{tabular}

\section{Perceptual Reproduction}

The difference between the actual distance of the target displacement and the perceptually estimated distance was calculated on each trial and used as a CE. Figure 4 shows the mean CEs and the VEs in Condition P. In this condition, the subjects showed a smaller constant directional bias than they did in the two motor-measure conditions. In all subjects, the CEs in the saccade conditions were more toward the positive direction (overestimation) than were the CEs in the pursuit-eye-movement conditions. The effect of eye-movement condition on CE was examined statistically in each subject, and shown to be significant for four subjects $[\mathrm{t}(67-97) \geq 6.57, \mathrm{p}<.01$, for Subjects A.K., T.K., and S.K.; $t(90)=2.64$, p < .05 , for Subject T.Y.). There was no significant difference in VEs between the two eye-movement conditions for any subjects.

\section{Comparison of the Three Response Conditions}

Figure 5 shows the difference in CEs between the two eye-movement conditions (CE in the saccade condition minus $C E$ in the pursuit-eye-movement condition) in each subject, separately for each response condition. In Condition $\mathbf{P}$, all subjects perceptually reproduced a longer distance of the target displacement in the saccade than in the pursuit-eye-movement conditions. A similar result was shown in Condition M1; all subjects pointed to positions farther from the fixation point in the saccade condition than in the pursuit-eye-movement condition. Note that, for all subjects, the size of the difference in CEs between the two eye-movement conditions was about the same in Conditions $\mathrm{P}$ and $\mathrm{M} 1$. On the other hand, there was a reduction in the size of the difference in CEs in Condition M2. Only two subjects showed significant differences in CEs between the two eye-movement conditions. Furthermore, when the subjects pointed to the target position from the right side, no differences were found for any of the subjects.

\section{Supplementary Experiment}

The mean CEs and VEs in pointing were calculated for each subject, and compared with those in Condition M2. In all subjects, the VEs in the supplementary experiment were significantly smaller than those in Condition $\mathbf{M} 2$. However, the mean CEs were about the same as those in Condition M2, and only one subject (Subject T.K.) showed a significant difference $[\mathrm{t}(29)=7.83, \mathrm{p}<.001$ Cochran-Cox test).

\section{DISCUSSION}

In Conditions M1 and M2, subjects showed a constant directional bias. The size and direction of this bias tended to be constant within each subject. Subjects A.K., S.K., and R.K. tended to overshoot the target, whereas Subjects T.Y. and T.K. had a tendency to undershoot the target. This directional bias made pointing accuracy ambiguous. For example, Subject A.K.'s pointing seems to be more accurate in the pursuit-eye-movement condition than

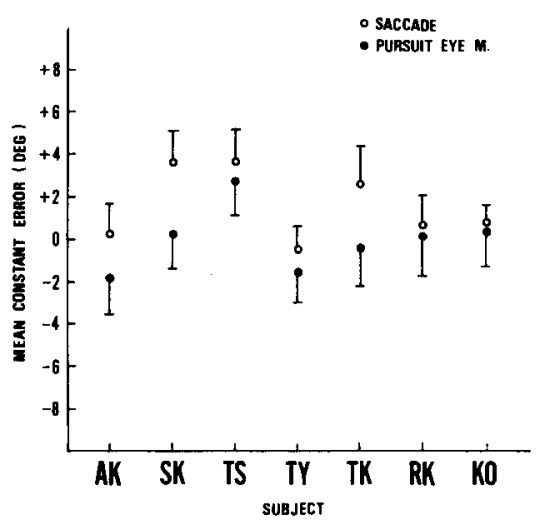

Figure 4. Mean constant error and variable error in Condition $P$. 

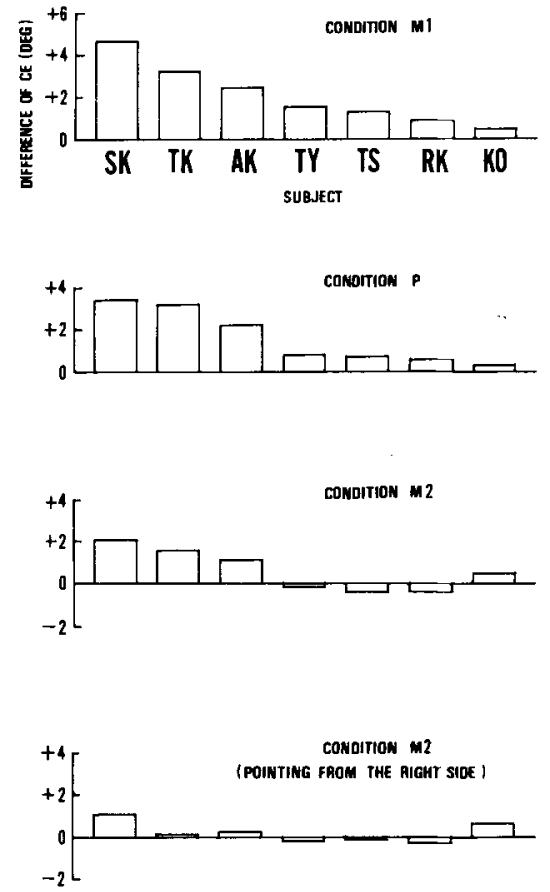

Figure 5. The difference in CEs between the two eye-movement conditions.

in the saccade condition, whereas Subject T.K.'s pointing seems to be more accurate in the saccade condition. Thus, it is impossible to decide in which eye-movement condition the subjects more accurately pointed to the target position. In a supplementary experiment, in which the locus of the constant directional bias was investigated, a target was presented in a structured visual field, and the subjects pointed to it with the unobservable hand. The result was that the mean CEs in pointing were about the same as those in Condition M2. This means that the subjects showed a constant directional bias even in this supplementary experiment, and that the directional bias shown in Conditions M1 and M2 was not due to the fact that because in these conditions the visual stimuli were presented in an unstructured visual field (i.e., in the total darkness), the subjects were not able to use visual context in judging the target position. It seems that the directional bias is due to an indigenous mismatch of information between the visual and the motor output systems. Therefore, the results of the supplementary experiment suggest that, in the saccade conditions of Conditions M1 and M2, the subjects accurately judged the target position and pointed to the position with an indigenous directional bias of the motor output system.

From this discussion, we can say that in the pursuiteye-movement condition of Condition M1, the subjects underestimated the distance of the target displacement, since, despite the above-mentioned directional bias, subjects' pointing shifted more toward the fixation point in the pursuit-eye-movement conditions than in the saccade conditions.
In Condition P, the estimated distance of the target displacement was about the same as its physical distance. Some subjects, however, showed a small directional bias. For each subject, the size and the direction of the bias were completely different from those found in motormeasure conditions, suggesting that the directional bias in Condition $\mathrm{P}$ is different in nature from that in Conditions M1 and M2. We think that the directional bias in Condition P was caused mainly by such factors in experimental method as a difference in light sources for target stimuli (laser beam) and for perceptual reproduction (LEDs), time lag between the offset of the target and the onset of the LEDs, a difference in the spatial position of the target and the LEDs, and so on. Because of the directional bias, there was no consistent tendency found in the differences between the two eye-movement conditions in accuracy of estimation, that is, in the absolute value of the CEs. All subjects, however, estimated the distance of the target displacement to be shorter in the pursuit-eyemovement conditions than in the saccade conditions. Therefore, if the directional bias is taken into account, the results of Condition P are consistent with the early finding that distance through which a displaced target appears to move is significantly shorter when pursuit tracked than when observed by means of a saccade (Mack \& Herman, 1972).

The main result of this study was that when the subjects pointed to the target from the left or the right side (Condition M2), the difference in CEs between the two eye-movement conditions disappeared. This was more prominent when the subjects pointed from the right side. As mentioned in the Method section, Condition M2 was introduced to examine the effect of perceptual factors in manual pointing in Condition M1. In the latter condition, the subjects pointed from the position of the fixation point. Therefore, the starting position and the direction of movement of the pointing hand were the same as those of the target movement. In this condition, the subjects were able to point to the target position by moving the pointing hand through the perceived extent of the target displacement. That is, they could point on the basis of the apparent distance of the target displacement. On the other hand, this was not the case in Condition M2. In particular, when the subjects pointed from the right side, both the starting position and the direction of movement of the pointing hand were different from those of the target movement. Thus, it is expected that pointing is more influenced by perceptual factors in Condition M1 than in Condition M2. Figure 5 shows that this expectation is correct. The pattern of differences in CEs between the two eye-movement conditions in Condition M1 was very similar to that in Condition P.

When the subjects' pointing was not influenced by the apparent distance of the target displacement, they pointed to about the same position in both the saccade and the pursuit-eye-movement conditions. This means that when a motor response is employed as a measure of EPS, spatial localization of the target position is as accurate in the 
pursuit-eye-movement conditions as it is in the saccade conditions. This is consistent with the finding that a fairly accurate EPS was shown during the pursuit-eye-movement condition when ballistic arm movement was used as a measure of EPS (Hansen, 1979; Hansen \& Skavenski, 1977).

The present study clearly indicated that there is a substantial difference between motor and perceptual measures. When the subjects perceptually judged the distance of the target displacement, they estimated it as shorter in the pursuit-eye-movement conditions than in the saccade conditions, but with manual pointing without perceptual contamination, the subjects located the target at about the same position in both eye-movement conditions. The discrepancy between perceptual and motor measures has been reported by many authors. Hansen (1979) showed that pointing an eye or limb to the location of a target seen during various types of eye movement is more accurate than verbal reporting of the same information. Bridgeman, Lewis, Heit, and Nagle (1979) showed that although subjects failed to detect a target displacement that occurred near the time of a saccade, they were still able to point to the center of the target with an unseen hand. They obtained a similar result in experiments using induced motions (Bridgeman, Kirch, \& Sperling, 1981). Mitrani, Dimitrov, Yakimoff, and Mateeff (1979) found that the position of the line of sight after the end of pursuit eye movement does not coincide with the perceived place of stimulus disappearance. These authors interpreted the discrepancy between perceptual and motor measures in terms of the two visual systems (Schneider, 1967; Treverthen, 1968). That is, perceptual and motor measures reflect the functions of the primary (retino-geniculo-striate pathway) and the secondary (retino-tectal pathway) visual systems, respectively. The present study shows, we think, that the second system receives information from the fovea, since even in the pursuit-eye-movement conditions in which the subjects continuously gazed at the visual target, and therefore the visual target was exclusively projected to the central area of the retina, the subjects were able to locate the target with the unobservable hand as accurately as they did in the saccade conditions.

\section{REFERENCES}

Bridgeman, B., Kirch, M., \& Sperling, A. (1981). Segregation of cognitive and motor aspects of visual function using induced motion. Perception \& Psychophysics, 29, 336-342.

Bridgeman, B., LeWIS, S., Heit, G., \& Nagle, M. (1979). Relation between cognitive and motor-oriented systems of visual position perception. Journal of Experimental Psychology: Human Perception and Performance, 5, 692-700.

Festinger, L., \& CANON, L. K. (1965). Information about spatial location based on knowledge about efference. Psychological Review, 72, 373-384.

Festinger, L., \& Easton, A. M. (1974). Inferences about the efference system based on a perceptual illusion produced by eye movements. Psychological Review, 81, 44-58.

HANSEN, R. M. (1979). Spatial localization during pursuit eye movements. Vision Research, 19, 1213-1221.

Hansen, R. M., \& Skavenski, A. A. (1977). Accuracy of eye position information for motor control. Vision Research, 17, 919-926.

Honda, H. (1984a). Eye position signals in successive saccades. Perception \& Psychophysics, 36, 15-20.

HONDA, H. (1984b). Functional between-hand differences and outflow eye position information. Quarterly Journal of Experimental Psychology, 36(A), 75-88.

Mack, A., \& Herman, E. (1972). A new illusion: The underestimation of distance during pursuit eye movements. Perception \& Psychophysics, 12, 471-473.

Mitrani, L., Dimitrov,G., Yakimoff, N., \& MateefF, S. (1979). Oculomotor and perceptual localization during smooth eye movements. Vision Research, 19, 609-612.

Rashbass, C. (1961). The relationship between saccadic and smooth tracking eye movements. Journal of Physiology (London), 159, 326-338.

SCHNEIDER, G. E. (1967). Contrasting visuomotor functions of tectum and cortex in the golden hamster. Psychologische Forschung, 31, $52-62$.

STOPER, A. E. (1973). Apparent motion of stimuli presented stroboscopically during pursuit movement of the eye. Perception \& Psychophysics, 13, 201-211.

TREVERTHEN, C. B. (1968). Two mechanisms of vision in primates. Psychologische Forschung, 31, 299-337.

(Manuscript received October 11, 1984; revision accepted for publication June 12, 1985.) 